Director, Monitoring and Research Branch, Department of Health, N.C.D.,

Papua New Guinea

\title{
A surveillance information system as a management tool: a report from Papua New Guinea
}

\section{Summary}

To have a very good surveillance system, it is paramount important to have a functional health information system that could be easily used for monitoring and investigation of disease outbreaks. In Papua New Guinea (PNG) a national health information system was developed, trailed and implemented nationwide. Furthermore to have the system working linked to it must be the local health system for sustainability and control. A public health manual for disease surveillance in PNG was developed and is now being used for surveillance.

This paper describes how the health information system, particularly surveillance system was developed and implemented on the national scale, how it was integrated with other management information systems and how information has been used to support management decision-making and informed policy decision. It will highlight some of the hurdles that it has encountered while trying to implement the system.

PNG has one of the best national health information system as compared to many developing countries but limited information generated from the system. There was also less feedback from all levels of the health sector. We need to improve surveillance on the basic principles of integration, focus, and sharing of work. There must be an appropriate and timely response and feedback. We need to improve on the current system rather than building a new one.

Keywords: Surveillance - Disease outbreaks - Papua New Guinea.

\section{The National Health Information System (NHIS)}

The NHIS was introduced nationwide in 1995 in PNG after a six months trail in Chimbu Province. Since then there was general acceptance of the system of which many health workers commented that it is less labor intensive and that the information collected was relevant. The system allows for active surveillance on notifiable and immunizable diseases in PNG. There is only one sheet of L4 size paper to send to provincial health officer rather than six or more different forms they used to sent in before the NHIS was introduced. They can now have access to their information at their provincial headquarters and furthermore can see how they perform as compared to other districts.

Data can be easily analyzed at national and local level using simple computer software, such as Microsoft Excel. The Minister and Secretary of Health can now confidently argue for more funds using the information generated through the NHIS. Many of the districts' health workers come to appreciate that they can do analysis on their local data that they never had done before. Many programme managers now have more access and more information to their programme than they did in the previous system.

\section{Health surveillance system as part of the PNG government reform}

PNG is prone to many epidemics. However these are not always recognized in the early stages, reported and are not always timely or adequately responded on. We had success in eradicating polio and eliminating leprosy, and measles because of the active sero-surveillance done at the national level and also filariasis has been targeted for elimination. We face challenges new in emerging diseases such as HIV/ AIDS, SARS and Avian Flue.

One of the main hindrances to effective surveillance is how the public health system is managed in PNG. The system was administered centrally after independence in 1975. All the programmes were highly vertical with the focus on the district level. The medical officers were the main force in the 
implementing of the programmes in the districts with strong support from the provincial health office and the hospital. The centralized system allowed for better management of the resources and ensured delivery of the services as planned. In that context health statistics collected nationally were useful for the centralized system because resource decisions were made at the national level.

After independence in 1977, the Organic Law on Provincial Government was introduced and legalized the decentralization of management and administration of all government services including health in the provinces. Under this Organic Law, the management and functions of health services were either transferred to or delegated to the provinces. The national functions were limited to policy and standards. There was poor definition of roles and responsibilities between the national and the provincial governments.

The national technical programme managers had insufficient and poor baseline health data from surveillance of notifiable diseases with little or no consultation with the provinces. There was no clear unified direction or leadership and this resulted in the development of uncoordinated plans and programmes by the provinces and the national department.

The collapse of the national health system started in the early 1980s. The Organic Law on Provincial Government allowed decentralization of resource management, but also brought lack of control and coordination, withdrawal of doctors from district health centres, the appointment of poorly trained and qualified provincial health managers, poor coordination between the provincial health office and the hospitals and lack of political will and commitment. The support to rural health from provincial health offices and hospitals gradually diminished and was eventually discontinued in the mid-80s due to the withdrawal of vehicles and funding. This contributed to a complete lack of direction, low staff morale, poor performance and a lack of community confidence in the health system. It also contributed to the uncoordinated collection of health statistics by health centres and hospitals. By the early 90s, the rural health system had come to a virtual standstill.
The new Organic Law on Provincial Governments and Local Level Governments, introduced in 1995, which called for effective delivery of government services to the rural population, was an attempt to rectify this situation. This allowed further decentralization to the districts with the focus of bringing development to the districts. The planning and implementation of the health programmes were initiated at the district level and funded by the District Budget Priority Committee, of which the local MP was to be chairman. The health sector in the district had to compete with other social sectors for the funds to run their programmes. This allowed for proper planning with facts and figures to secure the necessary resources. In order to do that the health facilities in the districts have to understand the use of their locally collected health statistics to do effective disease surveillance.

An other difficulty in setting up a surveillance system controlling diseases has been compounded by lack of standard protocols and management guidelines, and by the fact that front-line health workers have not been trained to deal with epidemics. Many of them do not know how to report, who to report to and when to report, and most importantly, what to do when there is an outbreak.

The Department of Health with the support from AusAid has developed a new manual "Public Health Manual for Papua New Guinea" (2002). This manual provides the necessary protocols and guidelines. It will be incorporated into in-service training and pre-service curricula for all levels of health workers. A training package has been developed to facilitate this. The manual contains the proposed strategy for improved surveillance of communicable diseases is a combination of the outpatient and peripheral facilities - outbreak reporting of any serious public health condition, syndromic reporting is encouraged in areas where there are no laboratories. The manual provides guidelines for hospital-based surveillance, public health programme and laboratory reporting. This is an ideal manual for surveillance; it is simple, flexible, acceptable, affordable and provides information that is sensitive, timely, representative and useful for public health action. This is an important skip towards a good health information system to continually collect health statistics for surveillance purpose.

\section{Reference}

AusAid (2002). Public health manual for Papua New Guinea: how to recognize, report and respond to epidemics: a anual for nurses, health extension officers and doctors. Port Moresby: Department of Health.

\section{Address for correspondence \\ Dr. Gilbert Hiawalyer (Director) \\ Monitoring and Research Branch \\ Department of Health \\ P.O. Box 807 \\ N.C.D \\ Papua New Guinea \\ Tel.: +675 301 3660/ 3013650 \\ Fax + 675 323002/3013604 \\ e-mail: ghiawaly@health.gov.pg}

